\title{
X-ray spectral and flux variability of the microquasar GRS 1758-258 on timescales from weeks to years
}

\author{
Maria Hirsch ${ }^{1}$, Katja Pottschmidt ${ }^{2,3}$, David M. Smith ${ }^{4}$, Arash Bodaghee ${ }^{5}$, Marion Cadolle Bel ${ }^{6}$, Victoria Grinberg ${ }^{7}$, \\ Natalie Hell ${ }^{8}$, Felicia Krauß ${ }^{9,10}$, Ingo Kreykenbohm ${ }^{1}$, Anne Lohfink ${ }^{11}$, Michael A. Nowak ${ }^{12}$, Bárbara H. Rodrigues ${ }^{13}$, \\ Roberto Soria ${ }^{14,15}$, John A. Tomsick ${ }^{16}$, and Jörn Wilms ${ }^{1}$
}

${ }^{1}$ Dr. Karl Remeis-Observatory \& ECAP, Universität Erlangen-Nürnberg, Sternwartstr. 7, 96049 Bamberg, Germany e-mail: maria.hirsch@fau.de

2 CRESST and NASA Goddard Space Flight Center, Greenbelt, MD 20771, USA

3 Department of Physics and Center for Space Science and Technology, University of Maryland, Baltimore, MD 21250, USA

4 Department of Physics and Santa Cruz Institute for Particle Physics, University of California, Santa Cruz, CA 95064, USA

5 Department of Chemistry, Physics and Astronomy, Georgia College and State University, Milledgeville, GA 31061, USA

6 Max Planck Computing and Data Facility, Gießenbachstr. 2, 85748 Garching, Germany

7 Institut für Astronomie und Astrophysik, Universität Tübingen, Sand 1, 72076 Tübingen, Germany

8 Lawrence Livermore National Laboratory, 7000 East Ave., Livermore, CA 94550, USA

9 GRAPPA \& Anton Pannekoek Institute for Astronomy, University of Amsterdam, Science Park 904, 1098 Amsterdam, The Netherlands

10 Department of Astronomy and Astrophysics, Pennsylvania State University, University Park, PA 16802, USA

11 Department of Physics, Montana State University, Bozeman, MT 59717-3840, USA

12 Department of Physics, Washington University, CB 1058, One Brookings Drive, St. Louis, MO 63130-4899, USA

13 Instituto Nacional de Pesquisas Espaciais, Avenida dos Astronautas, 1.758 São José dos Campos, Brazil

14 National Astronomical Observatories, Chinese Academy of Sciences, Beijing 100012, PR China

15 Sydney Institute for Astronomy, School of Physics A28, The University of Sydney, Sydney, NSW 2006, Australia

16 Space Sciences Laboratory, 7 Gauss Way, University of California, Berkeley, CA 94720-7450, USA

Received 15 November 2018 / Accepted 19 December 2019

\begin{abstract}
We present the spectral and timing evolution of the persistent black hole X-ray binary GRS 1758-258 based on almost 12 years of observations using the Rossi X-ray Timing Explorer Proportional Counter Array. While the source was predominantly found in the hard state during this time, it entered the thermally dominated soft state seven times. In the soft state GRS 1758-258 shows a strong decline in flux above $3 \mathrm{keV}$ rather than the pivoting flux around $10 \mathrm{keV}$ more commonly shown by black hole transients. In its 3-20 keV hardness intensity diagram, GRS 1758-258 shows a hysteresis of hard and soft state fluxes typical for transient sources in outburst. The RXTE-PCA and RXTE-ASM long-term light curves do not show any orbital modulations in the range of 2-30 d. However, in the dynamic power spectra significant peaks drift between 18.47 and $18.04 \mathrm{~d}$ for the PCA data, while less significant signatures between $19 \mathrm{~d}$ and $20 \mathrm{~d}$ are seen for the ASM data as well as for the Swift/BAT data. We discuss different models for the hysteresis behavior during state transitions as well as possibilities for the origin of the long term variation in the context of a warped accretion disk.
\end{abstract}

Key words. X-rays: binaries - X-rays: individuals: GRS 1758-258

\section{Introduction}

GRS $1758-258$ is a black hole binary discovered in 1990 during observations of the Galactic Center region by the Granat satellite (Mandrou 1990; Syunyaev et al. 1991, see Heindl \& Smith 2002 for the determination of the source's position). As one of only three persistent, mostly hard state, black hole binaries in our Galaxy and the Magellanic Clouds (GRS 1758-258, 1E 1740.7-2942, and Cyg X-1) ${ }^{1}$, GRS 1758-258 has since been observed in various energy ranges (e.g., Rodriguez et al. 1992; Cadolle Bel et al. 2006; Pottschmidt et al. 2008; Muñoz-Arjonilla et al. 2010; Soria et al. 2011; Luque-Escamilla et al. 2014, and references therein). As radio observations show a double-lobed

\footnotetext{
1 Other persistent black hole binaries are the Galactic source $4 \mathrm{U} 1957+11$, as well as LMC X-1 and LMC X-3, which are always or predominantly found in the soft state.
}

counterpart (Rodriguez et al. 1992) that shows similarities to winged radio galaxies (Martí et al. 2017), GRS 1758-258 is considered a microquasar.

The Rossi X-ray Timing Explorer (RXTE; Bradt et al. 1993) monitored GRS 1758-258 from 1996 to 2007 (see next section for a detailed description of these observations). Based on this program Smith et al. (2001) reported the transition to a soft state in 2001 during which the $3-25 \mathrm{keV}$ flux declined by more than an order of magnitude. This episode lasted around a year. Using XMM-Newton observations Soria et al. (2011) showed that this transition still followed the canonical evolution through states but with the soft state showing increased flux only below $\sim 3 \mathrm{keV}$. The occurrence of a less extended soft state in 2003 was reported by Pottschmidt et al. (2006).

Using the 2.5-25 keV RXTE PCA monitoring light curve of GRS 1758-258 from 1997 to 2002, Smith et al. (2002a) 
found a periodic signal of $18.45 \pm 0.10 \mathrm{~d}$. If this signal was due to a binary orbit and the companion is not a high-mass star (Martí et al. 1998), the companion would have to be a K giant star filling its Roche lobe (Rothstein et al. 2002). However, the identity of the companion star in the system was ambiguous for a long time (Smith 2010). After astrometric studies already hinted at the system being an intermediate-mass X-ray binary (Muñoz-Arjonilla et al. 2010; Luque-Escamilla et al. 2014), recent spectroscopy of the companion shows it is likely an A-type main sequence star (Martí et al. 2016). If true, the accretion process would still be Roche lobe overflow, which implies an orbital period in the range of 0.5-1.0 d (Martí et al. 2016), and, thus, significantly shorter than the signal at $18.45 \pm 0.10 \mathrm{~d}$ found by Smith et al. (2002a).

In order to better understand this puzzling source we took a closer look at the RXTE monitoring obervations of GRS 1758-258 for the first time spanning the full time range from 1996 to 2007. The observations and the Proportional Counter Array (PCA; Jahoda et al. 2006) data reduction are described in Sect. 2. In Sect. 3, we present the spectral analysis of the RXTE-PCA data of GRS 1758-258, starting with a description of how Galactic ridge emission was taken into account in Sect. 3.1 before defining the source model and overall spectral modeling procedure in Sect. 3.2, and adding the hardness intensity diagram (HID) of the dataset as an additional diagnostic in Sect. 3.3. The evolution of the spectral parameters as well as the HID show seven soft states during this time. In Sect. 4, we report on the timing analysis of the long-term light curve of GRS 1758-258, presenting periodograms of the raw as well as of the detrended RXTE-PCA light curves in Sects. 4.1 and 4.2. Finally, we also looked at periodograms of RXTE's All Sky Monitor (ASM; Levine et al. 1996) light curve of GRS 1758-258 in Sect. 4.3. We find no coherent periodic signal that could be identified as an orbital period in the range of $2 \mathrm{~d}$ to $30 \mathrm{~d}$, however, quasi-periodic oscillations are apparent in the period range around 18-20 d. In Sect. 5, we summarize and discuss the results and present our conclusions.

\section{Observations and data reduction}

RXTE monitored GRS 1758-258 with 1.0-1.5 ks long pointed snapshots starting in 1996 (Smith et al. 2001, 2002a). The exposures were performed in monthly intervals in 1996, weekly from 1997 through 2000, and twice a week from 2001 March to 2007 October. Each year there is a gap from November to January when the Sun was too close to the Galactic Center, that is the approximate pointing direction to GRS 1758-258.

The PCA consisted of five Proportional Counter Units (PCUs), each with a sensitivity between $2 \mathrm{keV}$ and $90 \mathrm{keV}$ with a field of view of $\sim 1^{\circ}$. The Proportional Counter Unit 2 (PCU2) was the best calibrated one (Jahoda et al. 2006). Since the top layer had the highest signal-to-noise ratio $(\mathrm{S} / \mathrm{N})$, we only used data from this layer of PCU2. We reduced the data with our standard analysis pipelines (Wilms et al. 1999, 2006) applying the NASA HEASARC software package HEASOFT, version 6.8 for the RXTE spectra ${ }^{2}$. Data up to $15 \mathrm{~min}$ after passage through the South Atlantic Anomaly (SAA) were excluded (Fürst et al. 2009). We also required an electron ratio below 0.5 in order to

\footnotetext{
2 As of the time of writing, HEASOFT had not changed in parts relevant for RXTE data reduction and calibration since this release. A difference of $0.4 \%$ in total flux for PCU2 with respect to later HEASOFT versions is due to an improved cross-calibration of the PCUs. This change does not affect our analysis.
}

exclude time periods of high background. We obtained observed spectra as well as instrumental background spectra using the "faint source" background model.

Because of its location only 0.66 away from the very bright X-ray binary GX 5-1, GRS 1758-258 was a difficult source to observe with the collimated detectors onboard RXTE. The monitoring could therefore only be realized using offset pointings away from GX 5-1 (Smith et al. 2001, 2002a), that is using the triangular response of PCA's collimator to reduce the influence of GX 5-1. Response matrices were built taking the effect of the offset pointings into account.

The spectral analysis was performed using data taken in standard2f mode, which provides 129 energy channels. The spectra were rebinned to a minimum $\mathrm{S} / \mathrm{N}$ of 5 . We used data in the energy range of 3-20 keV. All spectral fitting was done using the Interactive Spectral Interpretation System (ISIS; Houck \& Denicola 2000; Houck 2002; Noble \& Nowak 2008).

The PCA timing analysis was performed starting from the 3-20 keV fluxes of GRS 1758-258 determined for each monitoring observation in the spectral analysis. The ASM timing analysis was performed using the $3-5 \mathrm{keV}$ light curve ${ }^{3}$ available in NASA's HEASARC database in a daily binning for the same time range as the PCA light curve. Empty bins were removed. The Neil Gehrels Swift Observatory BAT 15-50 keV daily light curve was obtained from the BAT Transient Monitor (Krimm et al. 2013) ${ }^{4}$. Both the ASM and the BAT light curve do not require further processing before the analysis. Due to the location of GRS 1758-258 near the GX 5-1, MAXI (Matsuoka et al. 2009) cannot observe the source.

\section{Spectral evolution}

\subsection{Galactic ridge emission}

Because GRS 1758-258 is faint and located close to the Galactic center in the Galactic Plane, all RXTE spectra of the source also contain a strong, diffuse background component caused by the Galactic ridge emission in the X-rays. This emission has long been known to exist (Worrall et al. 1982; Warwick et al. 1985; Koyama et al. 1986); its origin, however, is still under discussion (Ebisawa et al. 2008; Warwick 2014; Nobukawa et al. 2016, and references therein). To distinguish between source counts and Galactic ridge counts, background observations totalling $13 \mathrm{ks}$, 1.5 offset from GRS 1758-258, were performed by RXTE in $1999^{5}$. We were able to model this local Galactic ridge Emission with two bremsstrahlung components and an iron line complex (see Fig. 1). As RXTE cannot resolve the individual iron line components, the position of the three lines was fixed to $6.4 \mathrm{keV}$, $6.67 \mathrm{keV}$, and $7.0 \mathrm{keV}$, respectively, with equivalent widths that scale as 85:458:129 according to CCD Suzaku observations of the Galactic ridge (Ebisawa et al. 2007). The normalization of the whole complex was left free to vary in the fit. The fit parameters are summarized in Table 1. We assume that there is no local variation of the Galactic ridge emission, and then, keeping all spectral parameters fixed at their best-fit values, added this model to the spectral model of GRS 1758-258. Figure 2 illustrates the contribution of the ridge emission to the measured spectrum.

\footnotetext{
ftp://legacy.gsfc.nasa.gov/xte/data/archive/ ASMProducts/definitive_1dwell/colors/xa_grs1758-258_ d1. col

4 https://swift.gsfc.nasa.gov/results/transients/ GRS1758-258/

5 ObsIDs 40097-09-01-00, 40097-09-02-00, 40097-09-02-01, 4009709-02-02, 40097-09-02-03, 40097-09-03-00, and 40097-09-04-00.
} 


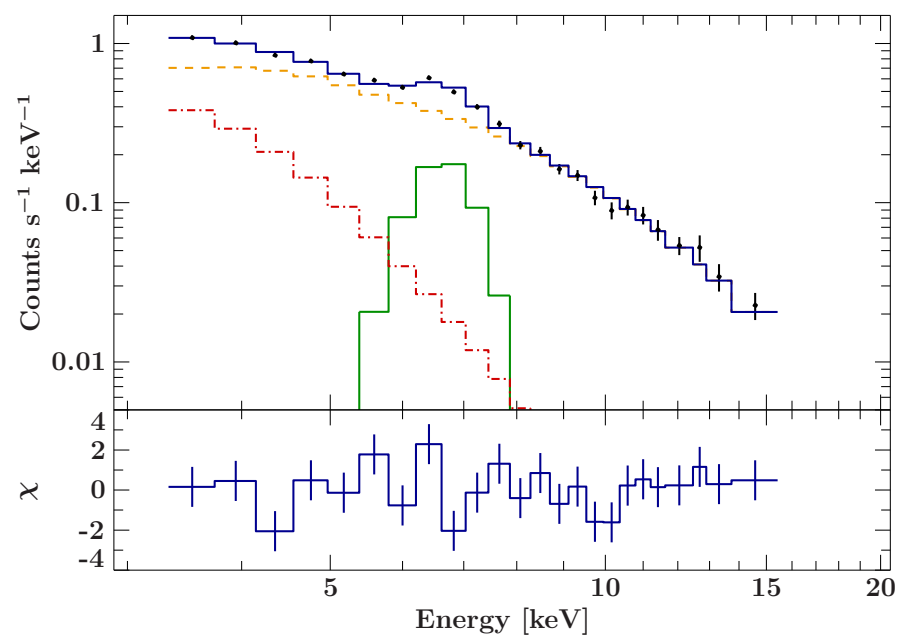

Fig. 1. Spectrum of the Galactic ridge emission as seen by RXTE. The data were fitted with two bremsstrahlung components (1: dashed line, 2: dash-dotted line) and an iron line complex as described in Ebisawa et al. (2007).

Table 1. Galactic ridge model parameters.

\begin{tabular}{lll}
\hline \hline$a_{\text {brems }, 1}$ & $0.011 \pm 0.003$ & \\
$k T_{1}$ & $8_{-1}^{+3}$ & $\mathrm{keV}$ \\
$a_{\text {brems }, 2}$ & $0.05_{-0.01}^{+0.03}$ & \\
$k T_{2}$ & $1.2_{-0.1}^{+0.2}$ & $\mathrm{keV}$ \\
\hline$F_{1}$ & $\left(2.6_{-0.4}^{+0.3}\right) \times 10^{-5}$ & $\mathrm{ph} \mathrm{s}^{-1} \mathrm{~cm}^{-2}$ \\
$\sigma_{1}$ & 0.05 & $\mathrm{keV}$ \\
$E_{1}$ & 6.4 & $\mathrm{keV}$ \\
$F_{2}$ & $1.4 \times 10^{-4}$ & $\mathrm{ph} \mathrm{s}^{-1} \mathrm{~cm}^{-2}$ \\
$\sigma_{2}$ & 0.05 & $\mathrm{keV}$ \\
$E_{2}$ & 6.67 & $\mathrm{keV}^{-1}$ \\
$F_{3}$ & $4 \times 10^{-5}$ & $\mathrm{ph} \mathrm{s} \mathrm{cm}^{-2}$ \\
$\sigma_{3}$ & 0.05 & $\mathrm{keV}$ \\
$E_{3}$ & 7.0 & $\mathrm{keV}$ \\
\hline
\end{tabular}

Notes. $a_{\text {brems }, 1,2}$ : normalization of the bremsstrahlung components; $k T_{1,2}$ : plasma temperature; $F_{1,2,3}$ is the total flux under a Gaussian centered at $E_{1,2,3}$ with a width $\sigma_{1,2,3}$. Values without uncertainties were kept fixed during the fit to the spectra from the background regions. Uncertainties are at the $90 \%$ confidence level.

\subsection{Spectral modelling}

Once the Galactic ridge background has been accounted for (see Sect. 3.1), all spectra were modeled using an empirical model consisting of an absorbed powerlaw (phabs $\times$ powerlaw). No high energy cutoff was needed as the cutoff energy is well above $20 \mathrm{keV}$ (Pottschmidt et al. 2006), the upper limit of the energy range considered here. The column density due to interstellar absorption in the direction of GRS 1758-258 was kept fixed to the canonical value of $N_{\mathrm{H}}=1.5 \times 10^{22} \mathrm{~cm}^{-2}$ (Mereghetti et al. 1997) using the abundances of Anders \& Grevesse (1989). Although the Galactic ridge spectral component already contains an iron line complex, the residuals show that this component is insufficient to explain the data in the $\mathrm{Fe} \mathrm{K} \alpha$ band. There are three potential explanations for such a deviation: the Galactic ridge emission could be spatially variable, there could be an intrinsic $\mathrm{Fe} \mathrm{K} \alpha$ emission or a combination of both effects.

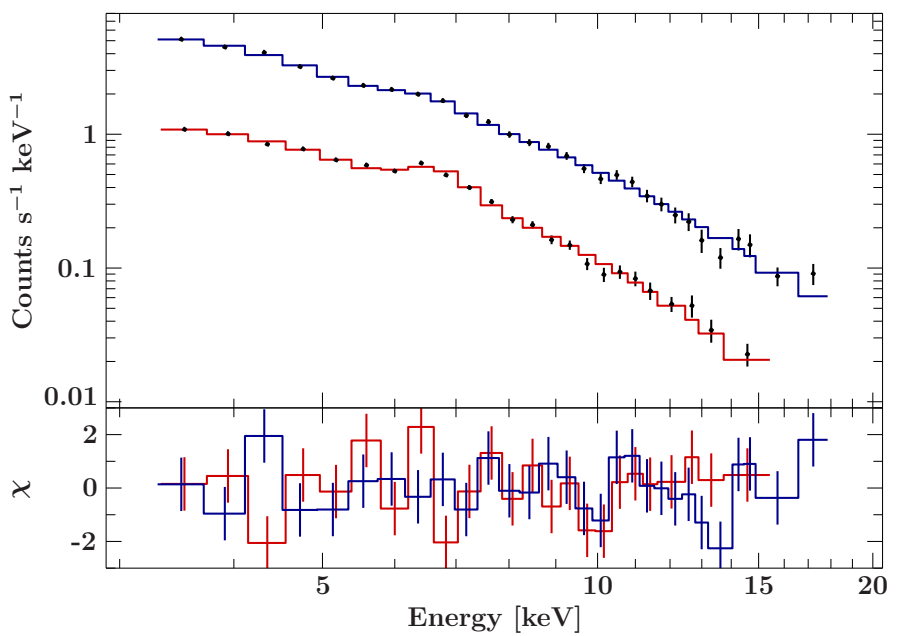

Fig. 2. RXTE-PCA spectrum of the total emission at the source position from the 2003 April observation. The total spectrum is modeled (blue histogram) as the sum of the source contribution and the Galactic ridge emission. The contribution of the Galactic ridge emission alone is also shown (red histogram).

In order to characterize the deviation of the observed line from the assumed (non-spatially varying) ridge component, we add a line at a fixed energy of $6.4 \mathrm{keV}$ to the model. We initially fixed the width of this line at $1 \mathrm{eV}$, well below the resolution of the PCA, and then fitted the flux of the line. In these fits a possible weak correlation between iron line flux and total source flux with a Spearman rank coefficient of 0.40 can be seen. A rough check of 100000 permutations of the iron line flux against the total source flux gives a mean rank coefficient of $7.9 \times 10^{-5}$ with individual values ranging between -0.15 and 0.15 . If true, this variability would indicate that part of the line would be source intrinsic. We note, however, that a narrow line at $6.4 \mathrm{keV}$ with a flux similar to that found in these fits would have been visible in the XMM-Newton observations discussed by Soria et al. (2011), but was not seen. RXTE's spectral resolution is so low, however, the width of the additional $\mathrm{Fe} \mathrm{K} \alpha$ is not well constrained: Re-fitting the RXTE spectra with the iron line width left as a free parameter results in an average line width of about $800 \mathrm{eV}$. Simulating XMM-Newton EPIC pn spectra for some of our best fit models with such a broad line smears out the iron line beyond recognition. For this reason we cannot claim that the XMM-Newton data formally rule out that some of the broad line flux originates in GRS 1758-258, although we consider this an astrophysically unlikely interpretation of the RXTE result.

Some softer spectra also require an additional disk blackbody for improving the fit. To estimate the significance of this improvement, we performed Monte Carlo simulations of the best fit model without the disk by creating a set of 1000 fake spectra for each observation. These synthetic spectra were then fitted with both models and the respective improvement in $\chi_{\text {red }}^{2}$ was calculated. We only accepted the disk component in our best fit model if the improvement in $\chi_{\text {red }}^{2}$ of the real dataset was above at least $99 \%$ of the fake spectra improvements. There are four occasions where a disk is detected at a very high temperature and low normalization. All four can be modeled with a higher normalization and lower temperature, with only slight worsening in the reduced $\chi^{2}$. These outliers thus are likely to be reflecting fit degeneracies and are not considered to be source intrinsic. 


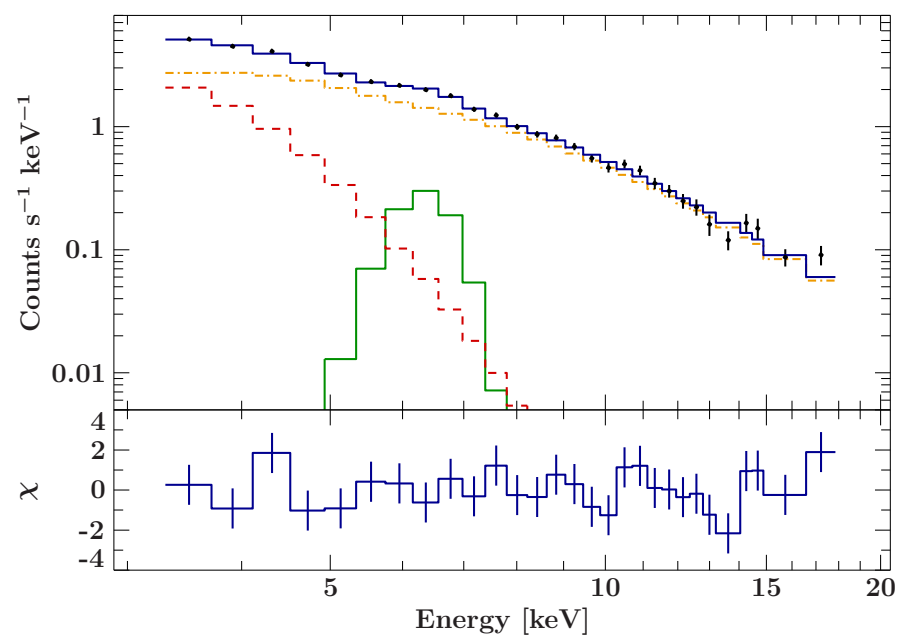

Fig. 3. Example of an instrument background subtracted spectrum taken by RXTE on 2009 April 08, containing the absorbed powerlaw component (dash-dotted line), the disk (dashed line), and the iron line (green solid line). For clarity the constant Galactic ridge model part is not shown.

Figure 3 shows an example of an instrument background subtracted spectrum and best fit model containing all the components.

To analyze the long term behavior of GRS 1758-258, for each spectrum, the source flux (i.e., with the Galactic ridge background subtracted but all other model components taken into account) was calculated integrating over the best fit model in the respective energy ranges for each observation. As apparent in Fig. 4, a change in flux is correlated with a change in the photon index: once the flux starts to decrease, the spectrum begins to soften. We classified as soft state all episodes that reach photon indices greater than two. The regions of interest were then defined to contain at least three data points before and after the peak, to fully catch the rise and decline. In some cases, this was not possible due to data gaps, which we do not want to cross for lack of information, for instance after the first soft state in 1999. Between 1997 and 2008, we found seven dim soft states, which are highlighted in Fig. 4. During the 2001 soft state, the source almost turned off completely with a remaining flux of only $\sim 0.045 \mathrm{keV} \mathrm{s}^{-1} \mathrm{~cm}^{-2}$ in the $3-20 \mathrm{keV}$ band. The blackbody disk emission appears only during these soft states where the low flux increases the uncertainties of the best fit parameters. Looking at the reduced $\chi^{2}$ (see Fig. 4, bottom panel), we find the fits slightly overdetermined, both for the fits with the fixed (Fig. 4) and the free iron line width. A further reduction of free parameters, however, is not possible: the Galactic ridge background is added as a constant with no free parameters ${ }^{6}$. The iron line position is fixed, as is the absorption towards GRS 1758-258 $\left(N_{\mathrm{H}}=1.5 \times 10^{22} \mathrm{~cm}^{-2}\right.$, Mereghetti et al. 1997). The only free model parameters are the powerlaw normalization and photon index, the iron line flux, and the disk flux and temperature where a disk black body is needed. Furthermore, we did not add a systematic uncertainty to the data. We therefore conclude that we can not improve on the overdetermination of our fits.

\footnotetext{
6 Variable ridge emission is in principle possible due to changes in roll angle and possible transient background sources, however, spectral modeling in which we kept the spectral shape of the ridge constant but let its flux vary does not lead to appreciable changes in the results presented in this paper compared to models where the ridge emission was kept fixed.
}

\subsection{Hardness intensity diagram}

As shown, for example, by Fender et al. (2004) or Belloni et al. (2005), it is typical for black hole transients to trace a q-shaped curve on their hardness intensity diagram (HID) during their outbursts. Pottschmidt et al. (2008) already found that GRS 1758-258 displays an unusual behavior in this respect: while persistent binaries usually occupy only a small area of the HID (see also Wilms et al. 2007), GRS 1758-258 shows a mixture of transient and persistent behavior. It moves anti-clockwise from the hard to the hard intermediate state, softens and then dims to the soft state, and finally hardens along the lower transitional branch back to the hard state. The 2001 extremely faint soft state directly follows an observational gap. Therefore, the transition from hard to soft state is not observed, leading to the atypical shape in the HID (Pottschmidt et al. 2008).

This behavior is confirmed here. Figure 5 shows the HID for the whole RXTE campaign. The soft states are highlighted using the same color scheme as in Fig. 4. It is obvious that GRS 1758-258 does not follow the usual q-shaped track but rather starts from the position of persistent sources on the upper right edge. Although the HID shows a clear hysteresis for hard and soft (absorbed) fluxes, there is no indication at all for a rise in the hard state from quiescence. No full return to the hard branch could be observed during the most extreme 2001 soft state: after the last soft state data point (MJD 52235.2961), there are no observations for almost two months. This mixed persistent/transient behavior of GRS 1758-258 was already observed by Smith et al. (2001), similar behavior has also been seen in 1E 1740.7-2942 (del Santo et al. 2005) and GX 339-4 (del Santo et al. 2008).

\section{Time series analysis}

For the time series analysis we used the complete flux light curve of GRS 1758-258, that is the sum of the flux band light curves shown in Fig. 4: as opposed to the count rate, flux values are independent of different detector responses to the respective spectral shape of GRS 1758-258. Due to many gaps and uneven spacing of the data points, we had to use the algorithm for generalized periodograms after Lomb (1976) and Scargle (1982).

\subsection{Flux light curve and its periodogram}

We now turn to the search for (quasi-)periodicities in the longterm light curve of the source, concentrating on the behavior of the $18.45 \pm 0.10 \mathrm{~d}$ periodicity found by Smith et al. (2002a) in the 1997-2001 RXTE-monitoring. Applying the Lomb-Scargle algorithm to the whole unfiltered flux light curve led to a power spectrum without any prominent peaks, and neither does the power spectrum calculated for the 1997-2001 data contain significant peaks (Fig. 6). The large luminosity variations between hard and soft spectral states can decrease the significance of period measurements and therefore cause the lack of such peaks (Smith et al. 2002a). In a next step we therefore perform a period search on de-trended data under consideration of possible systematic effects.

\subsection{Detrended flux light curve and its periodogram: a drifting 18 day period}

Excluding all soft states from the time series analysis would lead to major gaps in the light curve. In order to avoid any influence of the very dim and soft states on our periodogram, we only used 


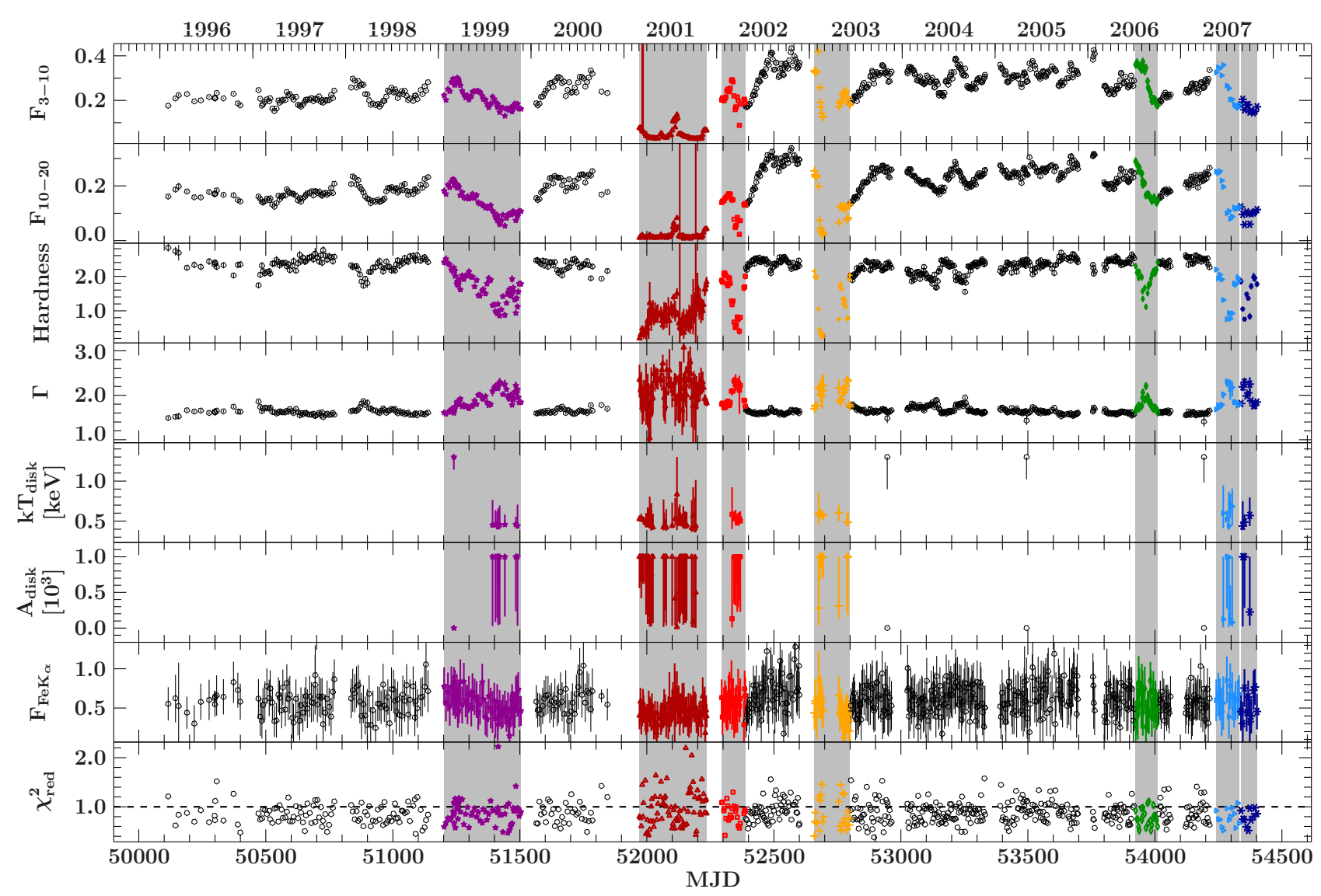

Fig. 4. Spectral parameters from RXTE monitoring observations of GRS1758-258: flux in $\mathrm{keV} \mathrm{s}^{-1} \mathrm{~cm}^{-2}$ in the 3-10 $\mathrm{keV}$ and $10-20 \mathrm{keV}$ bands, fitted to the spectra, spectral hardness (10-20 keV/3-5 keV) calculated with fluxes in $\mathrm{keV} \mathrm{s}^{-1} \mathrm{~cm}^{-2}$, photon index, temperature, and normalization of the disk component, total flux of the additional iron line in $10^{-3} \mathrm{ph} \mathrm{s}^{-1} \mathrm{~cm}^{-2}$ and the reduced $\chi^{2}$. Soft states are highlighted for episodes reaching a photon index greater than 2 .

data points with a photon index $\Gamma<2$ (see Sect. 3). This step alone is not sufficient, however, to remove long term variations and consequently no signal is seen in these power spectra. To avoid lower significances of our measurements caused by large luminosity variations and to be able to compare our results to those of Smith et al. (2002a), a high pass filter was then applied to the data by subtracting a smoothed version of the light curve. The following analysis uses this long-term trend and the high frequency residuals.

To generate the smoothed light curve, for each data point we fitted a straight line to all data within the range of $n=14 \mathrm{~d}$ before and after. The subsequent analysis only used the high frequency residual, that is the difference between the data point and the value of the straight line to obtain a high pass-filtered light curve. This method was already applied by Smith et al. (2002a), who used a range of $n=10 \mathrm{~d}$ before and after the data point. We extended this range in order to get better statistics for the smoothing fit, although we emphasize that our results do not depend on the exact value of $n$ chosen (Hirsch 2014). The smoothed long term trend and the residual flux light curve are shown in Fig. 7.

Using residual fluxes in the same time range as Smith et al. (2002a), that is 1997-2001, we are able to reproduce within the uncertainties the peak they found at $18.45 \pm 0.10 \mathrm{~d}$ (Fig. 7): although we did not exclude the low energy flux where no modulations are expected, we find a peak at $18.475 \pm 0.017 \mathrm{~d}^{7}$.

\footnotetext{
7 While Smith et al. (2002a) calculate their uncertainty using the FWHM of the peak in the PSD, we used 1000 sets of the long term
}

When using the whole 11 years of data, however, this peak is shifted in period by $0.32 \mathrm{~d}$ (Fig. 8 , left). Analyzing the data from 2002-2008, that is all data after the interval used by Smith et al. (2002a) and therefore statistically independent from their sample, this shift increases to $0.33 \mathrm{~d}$ (Fig. 8, right). This difference is reminiscent of changing superorbital modulations (see, e.g., Clarkson et al. 2003a,b). The figure also shows the maximum value of power spectra obtained when replacing the residual with Gaussian noise with the same mean and standard deviation. As discussed, for example, by Benlloch et al. (2001), these lines represent the "local significance" that the observed (quasi-) periodicity seen in the indicated $1 \mathrm{~d}$ broad period intervals is real. The Monte Carlo analysis automatically takes the trials factor into account. The power spectra therefore show significant peaks in the $18 \mathrm{~d}$ period band.

To study the evolution of the quasi periodic signal, we calculated a dynamic power spectrum (Fig. 9, see also Benlloch et al. 2001; Wilms et al. 2001): based on the 5 years interval of data originally used by Smith et al. (2002a), slices of the same length of 5 years were cut out of the light curve and analysed separately. Each time the starting time of the slice was shifted by $30 \mathrm{~d}$, and each resulting power spectrum is shown as a color-coded line in Fig. 9. Note that the 83 individual slices are overlapping and thus not statistically independent. As expected, the first few lines of

trend light curve plus Poisson-distributed random values for the respective light curve plus a sinusoid test signal at $16 \mathrm{~d}$. The standard deviation of the distribution of the PSD peaks of this test signal was then taken as a measure for our period uncertainty. 


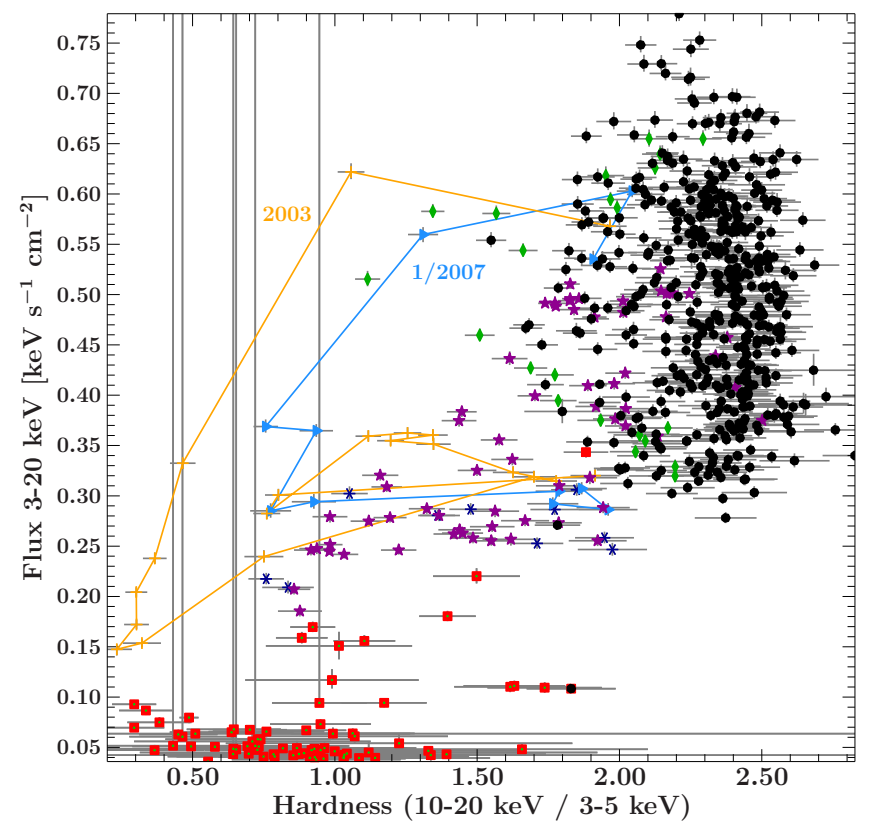

Fig. 5. Hardness intensity diagram (HID) from RXTE monitoring observations of GRS 1758-258 from 1997 until 2007. The seven dim soft states are highlighted as in Fig. 4. To show the "q"-shaped track of GRS 1758-258 in the HID, the data points of two soft state passages (2003 and 1/2007) are connected.

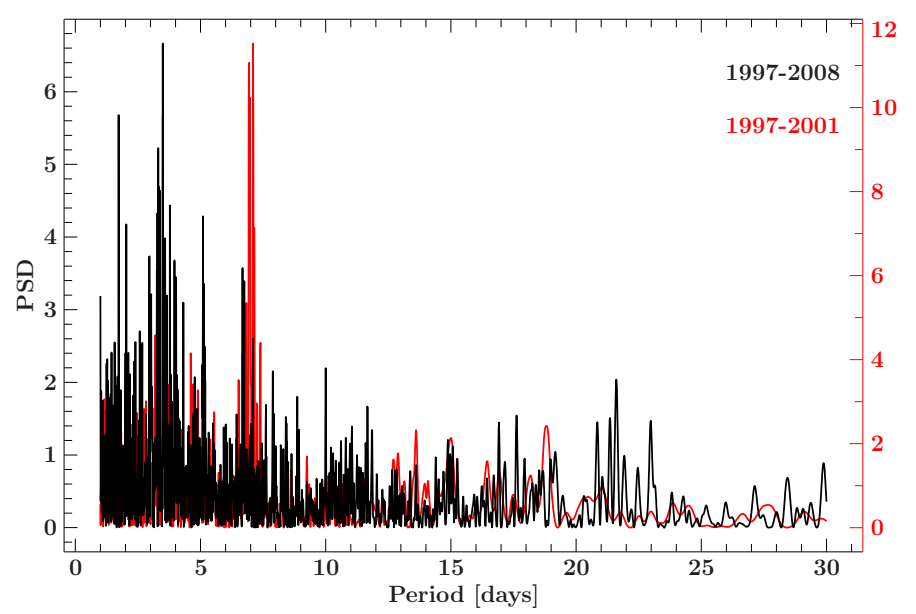

Fig. 6. Black: power spectrum of the full and unfiltered light curve, and red: power spectrum of the unfiltered 1997-2001 data already used by Smith et al. (2002a).

the dynamic periodogram show a peak at $18.475 \pm 0.017 \mathrm{~d}$. The maximum of this peak, however, is drifting with time, first to lower values to a minimum of $18.043 \pm 0.005 \mathrm{~d}$ in 2003 . Towards the end of the light curve, the period of the main peak is rising again. In addition, the period appears to fork into two peaks between 2000 and 2001. Figure 10 shows the modulation of the filtered light curve for the time range where the period in the dynamical power spectrum is stable (MJD 51769-53980).

Given the complexity of the data reduction, before we discuss the scientific implications of the peak we first discuss the significance of our measurements and explore how such a peak could be produced artificially.

We start by estimating the significance of the period. In order to do so we simulated 10000 light curves consisting of a white noise component, that is Gaussian distributed random values with the same standard deviation as that measured from the short term residuals obtained after detrending the original lightcurve. This approach therefore addresses both, the uncertainty of the individual flux measurements due to statistical effects as well as any excess noise that is due to intrinsic source variability. The residuals are consistent with Gaussian noise, such that more complex modeling, for example, using red noise residuals or applying a bootstrapping approach, is not necessary. For each simulated light curve, a dynamic power spectrum with 83 overlapping light curve slices was calculated in the same way as in Sect. 4.2. For each light curve slice, we then find the fraction of simulated white noise light curves that do not have their highest PSD peak in the range of the respective detected GRS 1758-258 period and its uncertainties. This fraction is a measure of the significance of the putative period of GRS 1758-258. We find significances for the drifting peak varying between $98.15 \%$ and $99.98 \%$.

Although our high pass filter works well for detecting periodic signals in the data, it is not ideal. For example, the long term trend light curve, which is subtracted by the filter, still contains part of periodic signals in the range of $14-25 \mathrm{~d}$. As the period found by Smith et al. (2002a) falls in this range, a closer look into the origin of this effect of the filter on the trend light curve is required.

To evaluate the range in which remnants of short-term periodic signals can be found in the long-term trend curve, we build an artificial light curve sampled at the same dates as those of the original light curve, containing Gaussian noise and a primary sinusoidal signal with a period in the range of 14-25 d. This light curve is then filtered to determine the long term trend. To test whether this trend still contains parts of the primary signal, we added an additional test signal at a different period, for example $12 \mathrm{~d}$, filtered again and applied the Lomb-Scargle technique as in Sect. 4.2. The resulting periodogram shows a main peak at the period of the test signal and a second peak at the period of the primary sinusoidal period. Since the analysis is based on a noise light curve, the residual signal is not connected to the flux values in our GRS 1758-258 light curve. Neither changing the period of the test signal nor changing the length of the light curve section we use has any influence on this effect. Randomly selecting two thirds of the data points in the light curve lessens the effect, and at the same time also reduces the power seen in the test signal peak.

The distribution of time intervals between individual observations of GRS 1758-258, that is the time intervals between the data points in the GRS 1758-258 light curve, does not show an excess for intervals between 14 and $25 \mathrm{~d}$. We can therefore exclude that the periodicity found in the long-term trend light curve is the result of the sampling of our GRS 1758-258 light curve. However, the range, in which the residual signal appears in the trend light curve, shifts according to the filter range $n$ and is always located between $n$ and $2 n$. Thus we conclude that this residual signal is left because the filter is not an ideal high pass filter. But as we find a significant peak in the periodogram although the filter removes part of the signal together with the long term trend, the filtering approach effect does not impair the main results of our analysis.

We also tested whether the drifting peak in the GRS 1758-258 dynamic power spectrum could be caused by the filtering process. In order to do so, we consider a pure white noise light curve with the original sampling, apply the high pass filter, and then calculate a dynamic power spectrum of the short term residuals analogous to the GRS 1758-258 dynamic power spectrum shown in Fig. 9. As expected, none 

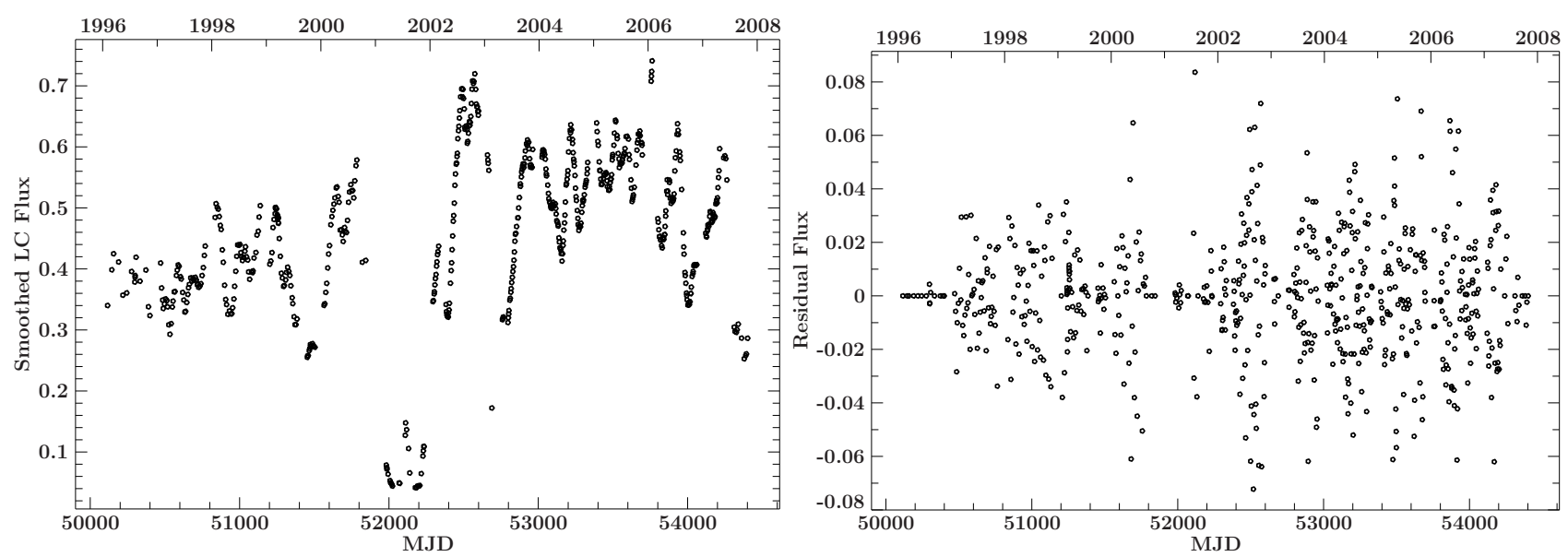

Fig. 7. Long term trend (left) and residual flux (right) light curves in $\mathrm{keV} \mathrm{s}^{-1} \mathrm{~cm}^{-2}$ after application of the high pass filter.
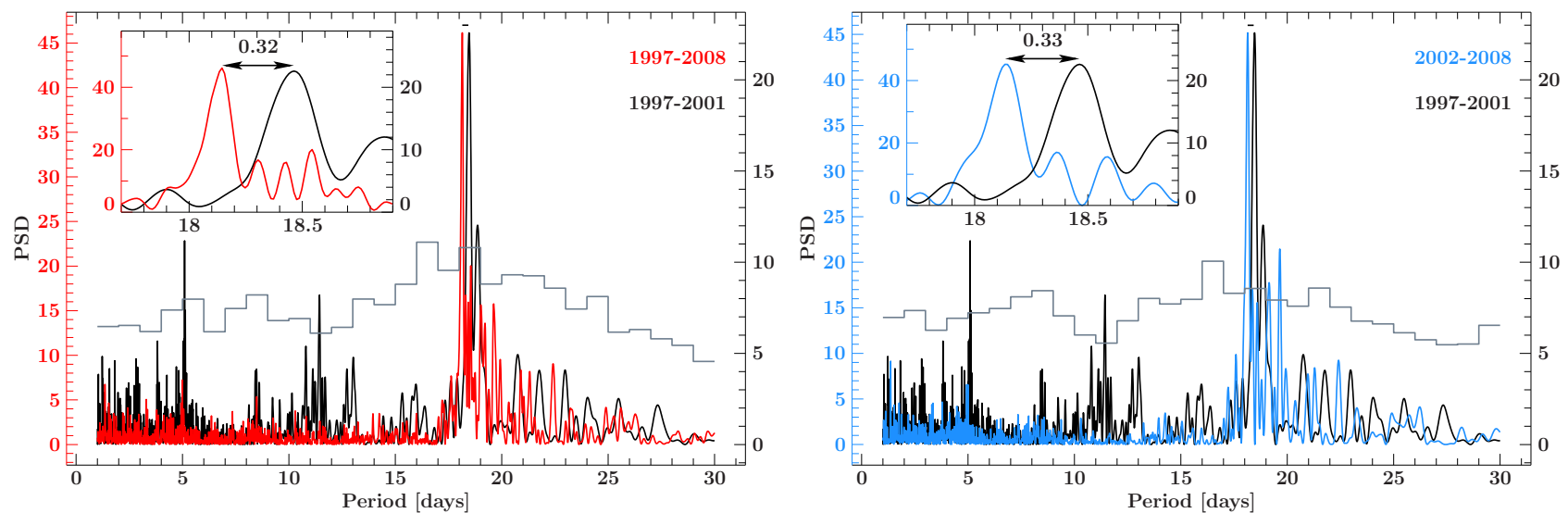

Fig. 8. Comparison of power spectra measured for the time interval 1997-2008 (red, left) and 2002-2008 (blue, right) to the power spectrum measured in the 1997-2001 data (black), i.e., the time interval used by Smith et al. (2002a). The peaks show a difference in period of $0.32 \mathrm{~d}$ and $0.33 \mathrm{~d}$, respectively. Histograms show the peak PSD values for 10000 realizations of a detrended light curve with residuals with a Gaussian distribution in the indicated period interval (see text for further explanation).

of the light curve slices, that is lines in the dynamic power spectrum, shows a prominent peak at periods between $1 \mathrm{~d}$ and $30 \mathrm{~d}$ in its periodogram. Thus, the period is not created by the filtering process.

Finally, in order to determine whether the shifting peak is real, we also tested whether the peak could be caused by the variability of the source being red noise. Red noise, used here to mean a stochastic process with a $f^{-\alpha}$ power spectrum, is notorious to exhibit quasi periodicities in its lightcurve when studying short light curve segments. We therefore used the algorithm of Timmer \& Koenig (1995) to generate a red noise lightcurve with 100000 data points (in order to avoid windowing effects caused by the simulation approach) with the same overall statistical properties as that of GRS 1758-258. We then selected a segment of that light curve to generate a red noise only lightcurve. This light curve was then analyzed in the same way as the real data, again using a high pass filter before applying the LombScargle algorithm. The resulting dynamic power spectrum does not show any peak at all in the period range we are interested in. We therefore can exclude a red noise origin for the drifting peak.

\subsection{Comparison with Levine et al. (2011)}

Smith et al. (2002a) were not the only ones reporting a periodicity for GRS 1758-258: Using ASM data in the 3-5 keV band and a different filtering approach, Levine et al. (2011) found a signal at a frequency of 0.0527 cycles day $^{-1}$, corresponding to a period of $18.97 \mathrm{~d}$. As this value is slightly different from what we obtain using the method of Smith et al. (2002a), we tried to reproduce their result, implementing the analysis method as described in Levine et al. (2011): we use the ASM light curve rebinned to a $2 \mathrm{~d}$ resolution and weighted according to the description in Levine et al. (2011), smooth it with a Gaussian kernel function with a full width at half maximum of $500 \mathrm{~d}$, and then calculate the power spectrum via the classical Fourier transform. This power spectrum is then whitened to account for background power. Detailed descriptions of all these steps can be found in the appendix of Levine et al. (2011). The reason for using a different approach than for the data described in Sect. 4.2 is twofold: first, it allows us to see whether the results of Levine et al. (2011) hold also for the longer time interval considered here. Secondly, using a different methodology on the ASM data set avoids introducing the same potential systematic errors in the analysis and thus allows an independent confirmation of the results of the PCA analysis of Sect. 4.2.

Using this method, we are able to roughly reproduce their power spectrum of GRS 1758-258 (Levine et al. 2011, their Fig. 9, bottom). There is a deviation in the total power in the power spectrum which is due to differences in the normalization of the Fourier transformation routines (Fig. 11). We then 


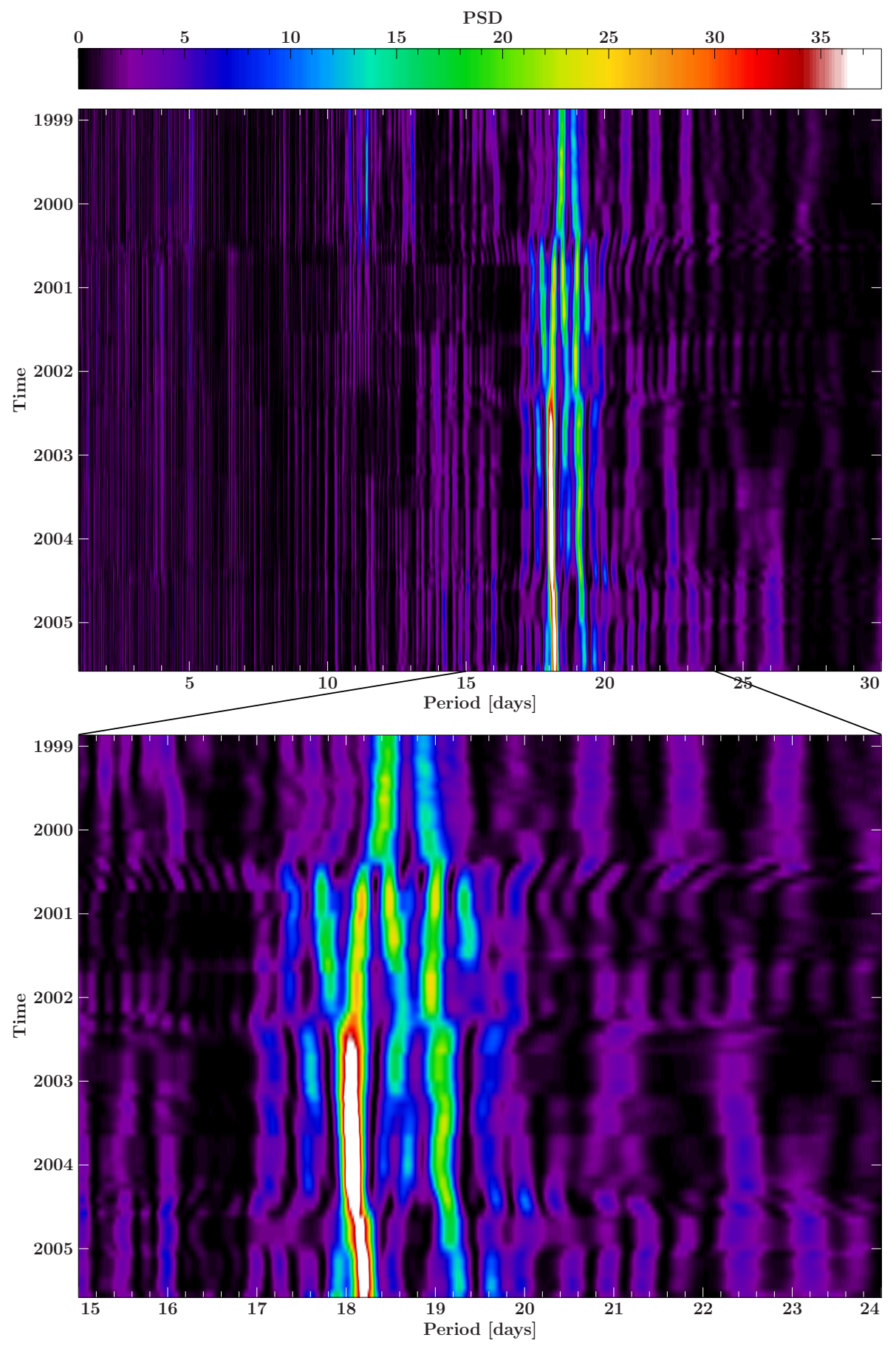

Fig. 9. Dynamical Lomb-Scargle periodogram for the entire 11 year $3-20 \mathrm{keV}$ model flux light curve of GRS 1758-258. The periodogram is calculated in five year intervals (centered on the middle in the Time axis), stepped in intervals of $30 \mathrm{~d}$. calculated a dynamic power spectrum (Fig. 12) for the same light curve slices as above, using the ASM 3-5 keV light curve binned on a $2 \mathrm{~d}$ grid and the analysis method of Levine et al. (2011). As the ASM data are noisier than the PCA flux light curve (Fig. 9), there is no prominent peak as seen in the PCA data. However, there is a feature around $19 \mathrm{~d}$ as reported by Levine et al. (2011). This feature blends with a peak that starts at a period of nearly $20 \mathrm{~d}$ and then drifts first toward smaller periods with a minimum in 2003. As with the PCA data and analysis after Smith et al. (2002a), a side peak showing a similar behavior at longer periods is also present. However, in the ASM data the side peak is at a distance of $\sim 2 \mathrm{~d}$ from the main peak, while in the PCA data the distance is only $\sim 1 \mathrm{~d}$. The region around mid-2000, where the main peak of the PCA data splits up into two peaks, is also interesting in the ASM data: the main peak drifts towards a minor peak and broadens as the two periods are close to each other.

On the whole, however, apart from a systematic discrepancy in periods of about $1 \mathrm{~d}$, the analysis of the ASM data using this different method confirms our first result of a drifting periodicity for GRS 1758-258: It is seen in different datasets, analyzed with different methods.

For another cross-check we analyzed the Swift/BAT light curve taken from the Transient Monitor (Krimm et al. 2013) following the algorithm of Smith et al. (2002a), using the same parameters for the high pass filter as in the analysis of the RXTE data. The resulting dynamic power spectrum is shown in Fig. 13. The energy band of $15-50 \mathrm{keV}$ also shows a drifting 


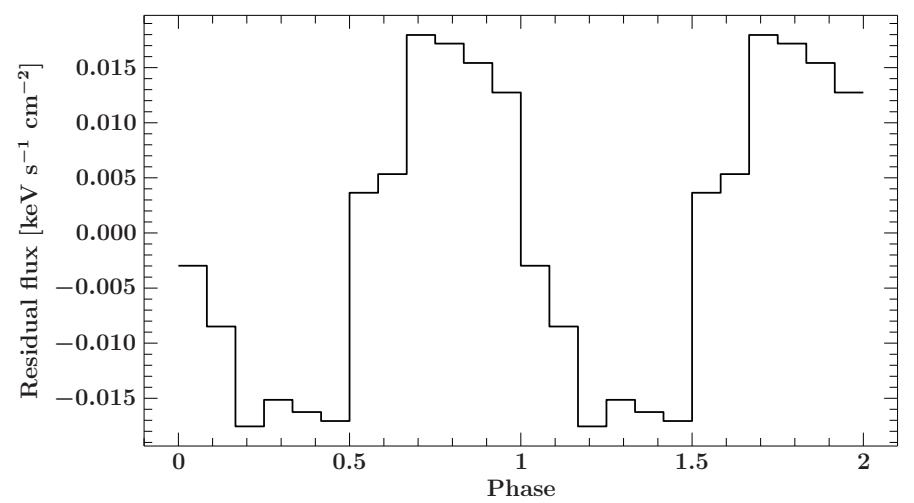

Fig. 10. Filtered 3-20 keV model flux light curve for the stable part of the dynamical power spectrum (MJD 51769-53980), folded on a period of $18.09 \mathrm{~d}$.

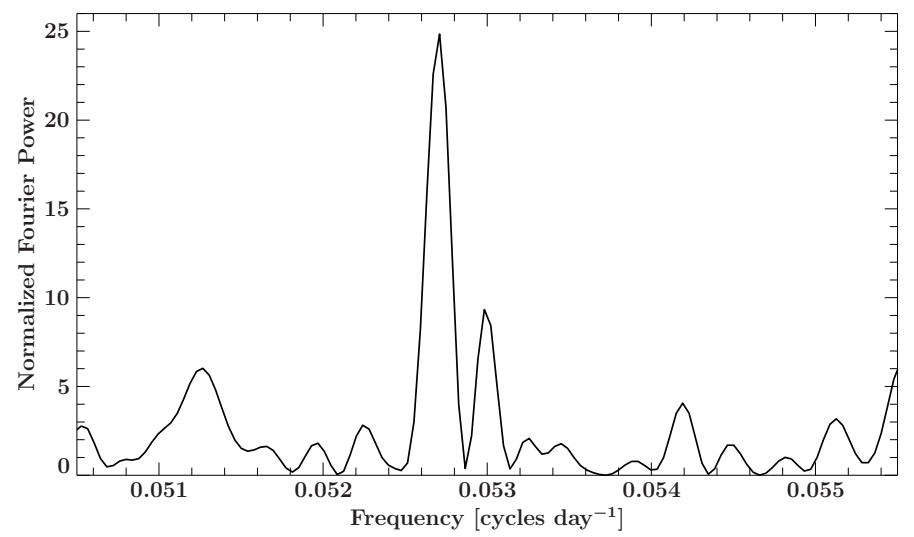

Fig. 11. Power spectrum of the $3-5 \mathrm{keV}$ ASM light curve, using the method of Levine et al. (2011). We are able to reproduce their Fig. 9 (bottom).

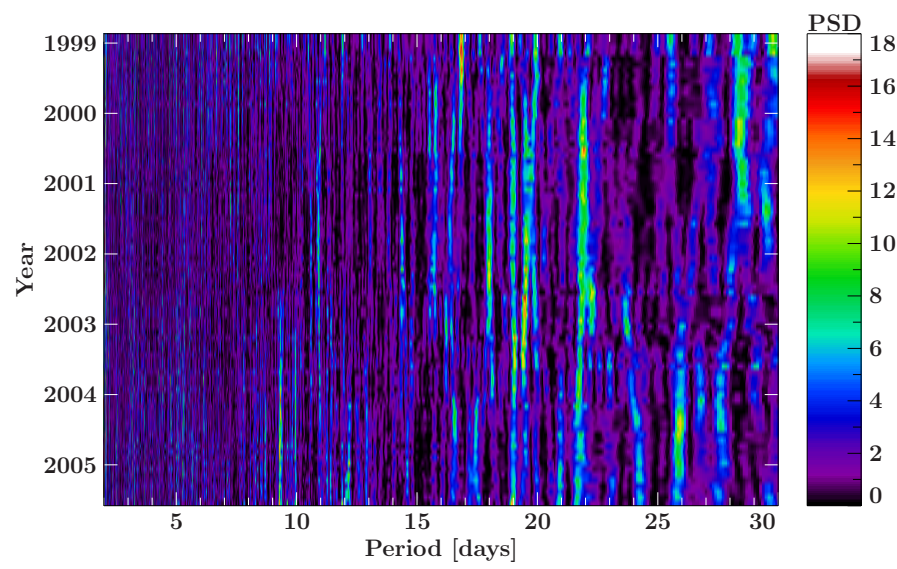

Fig. 12. Dynamic power spectrum of the $3-5 \mathrm{keV}$ ASM light curve, using the method of Levine et al. (2011). A drifting peak is visible although much less prominent due to the poor signal to noise ratio of the ASM data.

periodicity between $18 \mathrm{~d}$ and $19 \mathrm{~d}$. The signal is not as strong as in the PCA data, but the behavior is clearly the same in high as in low energies. Note that around the most recent, very dim 2016 soft state (Pottschmidt et al. 2016; Hirsch et al. 2016, and in prep.) a decrease in period is visible similar to the decrease in the PCA dynamic power spectrum around the very dim 2001 soft state. This additional observation of the drifting periodicity with another satellite than RXTE, at another time, in another

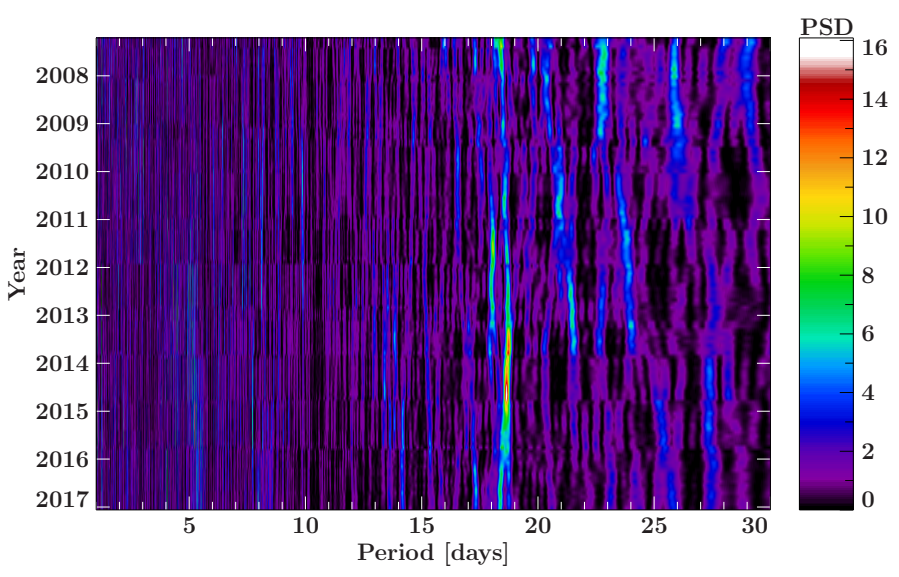

Fig. 13. Dynamic power spectrum of the $15-50 \mathrm{keV}$ Swift/BAT light curve, using the method of Smith et al. (2002a). The high energy light curve also shows a drifting peak. A change in period is obvious in 2016, near the most recent soft state.

energy range, further increases the confidence that we are seeing a source-intrinsic signal.

\section{Discussion}

\subsection{Spectral evolution and state transitions}

Analyzing data of the eleven years long RXTE-PCA monitoring of GRS $1758-258$ we find that the spectrum in the $3-20 \mathrm{keV}$ range can always be described by an absorbed powerlaw (photon indices varying between 1.5 and 3) with a neutral $\mathrm{Fe} \mathrm{K} \alpha$ line (which might be due to variations in the Galactic ridge emission) and, during the soft states with a photon index softer than 2, a disk-blackbody component. The Galactic ridge background emission was always accounted for. GRS 1758-258 entered a dim soft state for seven times between 1997 and 2008. During these soft states, the track of GRS 1758-258 in the hardness intensity diagram is similar to the q-shaped one of transient sources, however there is no rise from quiescence into the hard state. The high energy HID of GRS 1758-258 is comparable to that of Cyg X-1 (Obst et al. 2011).

The detection of an accretion disk in the soft state and its non-detection in the hard state are consistent with the $X M M$ Newton observations discussed by Soria et al. (2011), who also find a higher total X-ray luminosity in the 2001 XMM soft state data than in the 2008/2009 Swift XRT hard state observations. They conclude that luminosity cannot be the only driving force for a state transition in the GRS 1758-258 system.

There are several models that try to explain the evolution of $\mathrm{X}$-ray binaries in the HID. One of the first attempts to explain the hysteresis is the picture of two accretion flows set up and applied to GRS $1758-258$ by Smith et al. (2002b), and similar to models discussed by Meyer et al. (2000) and MeyerHofmeister et al. (2009): Based on Chakrabarti \& Titarchuk (1995), they suggest a Keplerian accretion disk in combination with a hot, sub-Keplerian halo accreting proportional amounts of matter. A boost in accretion rate leads to the halo brightening at once (almost free fall timescale), while the brightening of the inner regions of the disk is delayed by the inspiral of matter (viscous timescale). Then the additional soft photons are cooling the halo and the spectrum softens. Similarly, a sudden drop in accretion rate would first affect the halo, its Comptonizing component declining rapidly. The Keplerian disk reacts only on the viscous timescale, causing the soft component to decay 
slower (Smith et al. 2002b, 2007), which is the "dynamical soft state" observed in GRS 1758-258 and other black hole binaries, for instance, the transient source GX 339-4 (Debnath et al. 2015; Nagarkoti \& Chakrabarti 2016) and many other transients (Gierliński \& Newton 2006) as well as the persistent "twin source" of GRS 1758-258, 1E 1740.7-2942 (Smith et al. 2002b). Soria et al. (2011) refined this idea and suggest a magnetically powered coronal outflow as the source of the hard radiation. The accretion flow could then switch between the hard corona and the soft accretion disk because of changes in the poloidal magnetic field.

The interpretation of two accretion flows fits not only GRS 1758-258 but also other Galactic black hole binaries, With small changes, this picture can also be applied to high-mass X-ray binaries such as Cyg X-1 and LMC X-3 (Smith et al. 2002b, 2007): here, the mass input is no longer distributed proportionally between disk and halo. State changes are induced by the accretion flow switching between favouring the disk while starving the halo and favouring the halo while starving the disk. Thus, the bolometric luminosity should remain almost unchanged during state transitions in these systems. Recently, Ghosh \& Chakrabarti (2018) have found further evidence for an advective and a Keplerian flow analyzing time lags in the spectral slope for different high mass X-ray binaries and low mass X-ray binaries. They use a more extensive dataset and a completely different method than Smith et al. (2002a), yet their results lead to the same conclusion.

We note, however, that other models are equally successful at describing state transitions, such as the hybrid model of an outer standard accretion disk and an inner, magnetized jet emitting disk of Petrucci et al. (2008, see also Ferreira et al. 2006; Marcel et al. 2018a,b), the explanation of the hysteretic cycle in black hole state transitions as a magnetic field effect put forward by Begelman \& Armitage (2014), or the explanation of state transitions through severe disruptions of the accretion flow by Nixon \& Salvesen (2014). Common to all of these analyses and also to the large number of other discussions in the literature is that they are biased by the few bright and well sampled black hole outbursts such as those from GX 339-4 or XTE J1550-564, and that the more complex hysteretic behavior seen here or in other persistent sources such as LMC X-3 or Cyg X-1 is typically not explained. We hope that the data presented here will stimulate further theoretical discussions that address the difference of GRS 1758-258 and other black hole binaries.

\subsection{Timing behavior and long-term evolution}

Analyzing the model flux light curve spanning 11 years of observations for timing analysis, we are unable to detect any orbital modulation in the dataset. However, after detrending the data we find that the dynamic power spectrum exhibits a peak which drifts at periods between $18.475 \pm 0.017$ and $18.043 \pm 0.005 \mathrm{~d}$ and has a significance between $98.15 \%$ and $99.98 \%$. This drifting behavior was confirmed using another data set and analysis method, however with a systematic deviation in periods of about $2 \mathrm{~d}$.

Long-term periodicities in accreting systems are generally associated with periodic phenomena in the outer parts of the accretion disk, which are due to a combination of radiation pressure and orbital effects. The most prominent of such radiation driven periodicities are superhumps in cataclysmic variables (CVs). Such warps were first observed during superoutbursts of SU UMa systems (see Warner 2003, for a review and, e.g.,
Armstrong et al. 2013 for observations of superhumps in several CVs), where the superhumps are seen as periodic optical modulations caused by a 3:1 orbital resonance within the accretion disk, which causes the disk to be eccentric and to slowly precess. Here, irradiation of the accretion disk by the central source or inner part of the accretion disk results in a net torque on the disk which leads to a precessing, warped disk (Petterson 1977; Iping \& Petterson 1990; Pringle 1996; Wijers \& Pringle 1999; Maloney et al. 1996). The luminosity modulation is then caused by periodic variations of the efficiency of dissipative processes in the accretion disk (Whitehurst 1988; Whitehurst \& King 1991; Lubow 1991a,b).

Masetti et al. (1996), Haswell et al. (2001), and Charles (2002) review observations of superhumps in soft X-ray transients and low mass X-ray binaries. Masetti et al. (1996) suggest an alternative origin for these modulations: with an elliptical disk shape, the accretion flow impacts the outer disk at varying distances from the central object and thus at different gravitational potentials, leading to modulations in the released energy. Other possible mechanisms include a variation in the uncovered area in the direction of the observer or varying absorption by a disk warp. Based on this idea, Clarkson et al. (2003b) suggest a similar mechanism to explain the superorbital period of the high mass X-ray binary SMC X-1 (Wojdowski et al. 1998), which consists of a neutron star and the B0 I optical companion Sk 160 (Reynolds et al. 1993). Clarkson et al. (2003b) found this period to be varying between $40 \mathrm{~d}$ and $60 \mathrm{~d}$. These authors performed an analysis similar to ours, and also their dynamic power spectrum looks similar. They suggest the modulation being due to a bright spot at the intersection of accretion flow and accretion disk. This mechanism can support variations in the superorbital period (Clarkson et al. 2003b). In a follow-up paper, Clarkson et al. (2003a) present the analysis of a sample of other sources showing superorbital periods and put up a scheme, showing the evolution of disk warping with respect to the binary radius. With regard to the predictions of Ogilvie \& Dubus (2001), Clarkson et al. due to irradiation of the disk is impossible for very close binaries. With increasing separation of the binary components one stable warp mode as seen, for example, in Her X-1 or LMC X-4. Above the boundary region, several strong periodicities interact, as seen, for example, in Cyg X-2. In the border region itself, stable warping is not possible, as is seen in SMC $\mathrm{X}-1$, which shows sharp variations in the superorbital cycle length (Trowbridge et al. 2007). The underlying model, however, has to be more complex than described by Clarkson et al. (2003b), who expect a precessing warp and long periodicity for a source near this boundary, which is clearly inconsistent with the behavior reported by Trowbridge et al. (2007).

The result of Clarkson et al. (2003a,b) raises the question whether a similar mechanism is also applicable to GRS 1758-258. Both low mass X-ray binaries Her X-1 and LMC X-4 accrete via Roche lobe overflow, and the high mass X-ray binary SMC $\mathrm{X}-1$, too, is best characterized by Roche lobe overflow properties (e.g., Li \& van den Heuvel 1997; Içdem \& Baykal 2011). Given that the companion of GRS 1758-258 is probably an A-type star(Martí et al. 2016), the system would probably be somewhere in between Her X-1 or LMC X-4 and SMC X-1, such that the observed behavior is not fully unlikely.

Beyond that, superorbital periods have also been observed in wind-accreting high mass X-ray binaries (e.g., Corbet \& Krimm 2013). For such systems, different mechanisms have to be considered. Koenigsberger et al. (2006) suggest oscillations in the companion star driven by tidal interactions to be the source for the superorbital period, while Bozzo et al. (2017) propose 
corotating interaction regions in the stellar wind to be responsible for the observed modulations.

There are many mechanisms that lead to an observable variability in the light curve for different kinds of binary systems, and many aspects that can influence the formation of warps in an accretion disk for a system like GRS 1758-258, so that we cannot finally conclude this special mechanism of a warped disk to be the origin of the variable periodicity in GRS 1758-258.

\section{Conclusions}

Overall, the RXTE monitoring data show that GRS 1758-258 fits into the general picture of X-ray binaries with a few sourcecharacteristic features. The occasional very dim soft states as well as the striking timing behavior put a challenge to the current available physical models.

With the data currently available, it is neither possible to decide between the different models for the state transition in GRS 1758-258 nor between the mechanisms that lead to an observable variability in the light curve of a binary system. Further steps in theory and simulation have to be made to answer the open questions such as

- What physical model is behind the state transitions in GRS 1758-258 and other black hole binaries?

- Does the same model apply to low mass and high mass X-ray binaries, or do we need different mechanisms?

- Is there a model that can accomodate not only black hole binaries but also neutron star low-mass binaries, which display a similar behavior (Maccarone \& Coppi 2003; MuñozDarias et al. 2014)?

- What factors influence the formation of warps in accretion disks in GRS 1758-258 and other sources and how do they influence it?

- Is it possible to explain both effects in one comprehensive picture?

Especially for the last two items, further observations of systems that are displaying superorbital periods are needed to have a statistical relevant sample of different behaviors and to be able to fit in the scheme sources as GRS 1758-258 where we do not know much about the distance, the companion or the binary separation and orbit.

Acknowledgements. This research has made use of ISIS functions provided by ECAP/Remeis observatory and MIT (http://www. sternwarte uni-erlangen.de/isis/). We thank John E. Davis for the development of the SLxfig module, which was used to create all figures in the paper. This research was partially funded by the Bundesministerium für Wirtschaft und Technologie under Deutsches Zentrum für Luft- und Raumfahrt grant 50 OR 1113. This work has been partially funded by the European Commission through grant ITN 215212 "Black Hole Universe". It was partially completed by LLNL under the auspices of the US DOE under Contract DE-AC52-07NA27344. We acknowledge the support by the DFG Cluster of Excellence "Origin and Structure of the Universe" and are grateful for the support by MCB through the Computational Center for Particle and Astrophysics (C2PAP). Support for this work was provided by NASA through the Smithsonian Astrophysical Observatory (SAO) contract SV3-73016 to MIT for Support of the Chandra X-Ray Center (CXC) and Science Instruments. CXC is operated by SAO for and on behalf of NASA under contract NAS8-03060. F. K. acknowledges funding from the WARP program of The Netherlands Organisation for Scientific Research (NWO) unde grant agreement No. 648.003.002, and was supported as an Eberly Research Fellow by the Eberly College of Science at the Pennsylvania State University. V. G. is supported through the Margarete von Wrangell fellowship by the ESF and the Ministry of Science, Research and the Arts Baden-Württemberg.

\section{References}

Anders, E., \& Grevesse, N. 1989, Geochim. Cosmochim. Acta, 53, 197 Armstrong, E., Patterson, J., Michelsen, E., et al. 2013, MNRAS, 435, 707
Begelman, M. C., \& Armitage, P. J. 2014, ApJ, 782, L18

Belloni, T., Homan, J., Casella, P., et al. 2005, A\&A, 440, 207

Benlloch, S., Wilms, J., Staubert, R., \& Nowak, M. 2001, in Exploring the Gamma-Ray Universe, eds. A. Gimenez, V. Reglero, \& C. Winkler (Noordwijk: ESA Publications Division), ESA Publ. Division, 459, 263

Bozzo, E., Oskinova, L., Lobel, A., \& Hamann, W.-R. 2017, A\&A, 606, L10 Bradt, H. V., Rothschild, R. E., \& Swank, J. H. 1993, A\&AS, 97, 355

Cadolle Bel, M., Goldwurm, A., \& Sizun, P. 2006, in Populations of High Energy Sources in Galaxies, eds. E. J. A. Meurs, \& G. Fabbiano (Cambridge: Cambridge University Press), IAU Symp., 230, 93

Chakrabarti, S., \& Titarchuk, L. G. 1995, ApJ, 455, 623

Charles, P. A. 2002, in The Physics of Cataclysmic Variables and Related Objects, eds. B. T. Gänsicke, K. Beuermann, \& K. Reinsch (San Francisco: ASP), ASP Conf. Ser., 261, 223

Clarkson, W. I., Charles, P. A., Coe, M. J., \& Laycock, S. 2003a, MNRAS, 343, 1213

Clarkson, W. I., Charles, P. A., Coe, M. J., et al. 2003b, MNRAS, 339, 447

Corbet, R. H. D., \& Krimm, H. A. 2013, ApJ, 778, 45

Debnath, D., Mondal, S., \& Chakrabarti, S. K. 2015, MNRAS, 447, 1984

del Santo, M., Bazzano, A., Zdziarski, A. A., et al. 2005, A\&A, 433, 613

del Santo, M., Malzac, J., Jourdain, E., Belloni, T., \& Ubertini, P. 2008, MNRAS, 390,227

Ebisawa, K., Yamauchi, S., Tanaka, Y., Koyama, K., \& Suzaku Team 2007, Theor. Phys. Suppl., 169, 121

Ebisawa, K., Yamauchi, S., Tanaka, Y., et al. 2008, PASJ, 60, 223

Fender, R. P., Belloni, T. M., \& Gallo, E. 2004, MNRAS, 355, 1105

Ferreira, J., Petrucci, P.-O., Henri, G., Saugé, L., \& Pelletier, G. 2006, A\&A, 447,813

Fürst, F., Wilms, J., Rothschild, R. E., et al. 2009, Earth Planet. Sci. Lett., 281, 125

Ghosh, A., \& Chakrabarti, S. K. 2018, MNRAS, 479, 1210

Gierliński, M., \& Newton, J. 2006, MNRAS, 370, 837

Haswell, C. A., King, A. R., Murray, J. R., \& Charles, P. A. 2001, MNRAS, 321, 475

Heindl, W. A., \& Smith, D. M. 2002, ApJ, 578, L125

Hirsch, M. 2014, Master's Thesis, Remeis-Observatory, Friedrich-AlexanderUniversität Erlangen-Nürnberg, Bamberg, Germany

Hirsch, M., Pottschmidt, K., Krauss, F., et al. 2016, ATel, 9890

Houck, J. C. 2002, in High Resolution X-ray Spectroscopy with XMM-Newton and Chandra, ed. G. Branduardi-Raymont, Proc. 2002 MSSL Workshop

Houck, J. C., \& Denicola, L. A. 2000, in Astronomical Data Analysis Software and Systems IX, eds. N. Manset, C. Veillet, \& D. Crabtree (San Francisco: ASP), ASP Conf. Ser., 216, 591

Içdem, B., \& Baykal, A. 2011, A\&A, 529, A7

Iping, R. C., \& Petterson, J. A. 1990, A\&A, 239, 221

Jahoda, K., Markwardt, C. B., Radeva, Y., et al. 2006, ApJS, 163, 401

Koenigsberger, G., Georgiev, L., Moreno, E., et al. 2006, A\&A, 458, 513

Koyama, K., Makishima, K., Tanaka, Y., \& Tsunemi, H. 1986, PASJ, 38, 121

Krimm, H. A., Holland, S. T., Corbet, R. H. D., et al. 2013, ApJS, 209, 14

Levine, A. M., Bradt, H., Cui, W., et al. 1996, ApJ, 469, L33

Levine, A. M., Bradt, H. V., Chakrabarty, D., Corbet, R. H. D., \& Harris, R. J. 2011, ApJ, 196, 6

Li, X.-D., \& van den Heuvel, E. P. J. 1997, A\&A, 321, L25

Lomb, N. R. 1976, Ap\&SS, 39, 447

Lubow, S. H. 1991a, ApJ, 381, 259

Lubow, S. H. 1991b, ApJ, 381, 268

Luque-Escamilla, P. L., Martí, J., \& Muñoz-Arjonilla, Á. J. 2014, ApJ, 797, L1

Maccarone, T. J., \& Coppi, P. S. 2003, MNRAS, 338, 189

Maloney, P. R., Begelman, M. C., \& Pringle, J. E. 1996, ApJ, 472, 582

Mandrou, P. 1990, IAU Circ., 5032

Marcel, G., Ferreira, J., Petrucci, P.-O., et al. 2018a, A\&A, 617, A46

Marcel, G., Ferreira, J., Petrucci, P. O., et al. 2018b, A\&A, 615, A57

Martí, J., Mereghetti, S., Chaty, S., et al. 1998, A\&A, 338, L95

Martí, J., Luque-Escamilla, P. L., \& Muñoz-Arjonilla, Á. J. 2016, A\&A, 596, 46

Martí, J., Luque-Escamilla, P. L., Bosch-Ramon, V., \& Paredes, J. M. 2017, Nat. Commun., 8, 1757

Masetti, N., Bianchini, A., Bonibaker, J., della Valle, M., \& Vio, R., 1996, A\&A, 314,123

Matsuoka, M., Kawasaki, K., Ueno, S., et al. 2009, PASJ, 61, 999

Mereghetti, S., Cremonesi, D. I., Haardt, F., et al. 1997, ApJ, 476, 829

Meyer, F., Liu, B. F., \& Meyer-Hofmeister, E. 2000, A\&A, 361, 175

Meyer-Hofmeister, E., Liu, B. F., \& Meyer, F. 2009, A\&A, 508, 329

Muñoz-Arjonilla, A. J., Martí, J., Luque-Escamilla, P. L., et al. 2010, A\&A, 519, A15

Muñoz-Darias, T., Fender, R. P., Motta, S. E., \& Belloni, T. M. 2014, MNRAS, 443, 3270

Nagarkoti, S., \& Chakrabarti, S. K. 2016, MNRAS, 462, 850

Nixon, C., \& Salvesen, G. 2014, MNRAS, 437, 3994 
Noble, M. S., \& Nowak, M. A. 2008, PASP, 120, 821

Nobukawa, M., Uchiyama, H., Nobukawa, K. K., Yamauchi, S., \& Koyama, K. 2016, ApJ, 833, 268

Obst, M., Pottschmidt, K., Lohfink, A., et al. 2011, Acta Polytech., 51, 49

Ogilvie, G. I., \& Dubus, G. 2001, MNRAS, 320, 485

Petrucci, P.-O., Ferreira, J., Henri, G., \& Pelletier, G. 2008, MNRAS, 385 , L88

Petterson, J. A. 1977, ApJ, 216, 827

Pottschmidt, K., Chernyakova, M., Zdziarski, A. A., et al. 2006, in The X-ray Universe 2005, ed. A. Wilson, ESA Spec. Publ., 604, 283

Pottschmidt, K., Chernyakova, M., Lubiński, P., et al. 2008, Proceedings of "An INTEGRAL view of compact objects" - 7th INTEGRAL Workshop (PoS[Integral08]), 67

Pottschmidt, K., Eikmann, W., Kreykenbohm, I., et al. 2016, ATel, 9625

Pringle, J. E. 1996, MNRAS, 281, 357

Reynolds, A. P., Hilditch, R. W., Bell, S. A., \& Hill, G. 1993, MNRAS, 261, 337

Rodriguez, L. F., Mirabel, I. F., \& Marti, J. 1992, ApJ, 401, L15

Rothstein, D. M., Eikenberry, S. S., Chatterjee, S., et al. 2002, ApJ, 580, L61

Scargle, J. D. 1982, ApJ, 263, 835

Smith, D. M., Heindl, W. A., Markwardt, C. B., \& Swank, J. H. 2001, ApJ, 554, L41

Smith, D. M., Heindl, W. A., \& Swank, J. H. 2002a, ApJ, 578, L129

Smith, D. M., Heindl, W. A., \& Swank, J. H. 2002b, ApJ, 569, 362

Smith, D. M., Dawson, D. M., \& Swank, J. H. 2007, ApJ, 669, 1138
Smith, I. A. 2010, in AAS/High Energy Astrophysics Division \#11, Bull. Am. Astron. Soc., 42, 671

Soria, R., Broderick, J. W., Hao, J., et al. 2011, MNRAS, 415, 410

Syunyaev, R., Gilfanov, M., Churazov, E., et al. 1991, Sov. Astron. Lett., 17, 50 Timmer, J., \& Koenig, M. 1995, A\&A, 300, 707

Trowbridge, S., Nowak, M. A., \& Wilms, J. 2007, ApJ, 670, 624

Warner, B. 2003, Cataclysmic Variable Stars (Cambridge: Cambridge University Press)

Warwick, R. S. 2014, MNRAS, 445, 66

Warwick, R. S., Turner, M. J. L., Watson, M. G., \& Willingale, R. 1985, Nature, 317,218

Whitehurst, R. 1988, MNRAS, 232, 35

Whitehurst, R., \& King, A. 1991, MNRAS, 249, 25

Wijers, R. A. M. J., \& Pringle, J. E. 1999, MNRAS, 308, 207

Wilms, J., Nowak, M. A., Dove, J. B., Fender, R. P., \& Di Matteo, T. 1999, ApJ, 522,460

Wilms, J., Nowak, M. A., Pottschmidt, K., et al. 2001, MNRAS, 320, 327

Wilms, J., Nowak, M. A., Pottschmidt, K., Pooley, G. G., \& Fritz, S. 2006, A\&A, 447,245

Wilms, J., Pottschmidt, K., Pooley, G. G., et al. 2007, ApJ, 663, L97

Wojdowski, P., Clark, G. W., Levine, A. M., Woo, J. W., \& Zhang, S. N. 1998 ApJ, 502, 253

Worrall, D. M., Marshall, F. E., Boldt, E. A., \& Swank, J. H. 1982, ApJ, 255, 111 